\title{
Cytoprotective effects of urinary trypsin inhibitor on astrocytes injured by sustained compression
}

\author{
Shuang Zhang $\cdot$ Rongguo Yu • Yingrui Zhang • \\ Kai Chen
}

Received: 14 September 2012/ Accepted: 24 December 2013/Published online: 3 January 2014

(C) The Author(s) 2014. This article is published with open access at Springerlink.com

\begin{abstract}
Decreased cell membrane integrity is a primary pathological change observed in traumatic brain injury (TBI) that activates a number of complex intercellular and intracellular pathological events, leading to further neural injury. In this paper, we assessed the effects of urinary trypsin inhibitor (UTI) on astrocyte membrane integrity by determining the percentage of lactate dehydrogenase $(\mathrm{LDH})$ released after sustained compression injury using a hydrostatic pressure model of mechanical-like TBI. Astrocytes isolated from SD rat pups were injured by sustained compression. At a pressure of $0.3 \mathrm{MPa}$ for $5 \mathrm{~min}$, a significant increase in LDH release was observed compared with control samples. Astrocytes displayed extensive structural disruption of mitochondrial cristae reflected in their swelling. Based on our initial results, injured astrocytes were treated with UTI at a final concentration of 500, $1,000,3,000$ or $5,000 \mathrm{U} / \mathrm{ml}$ for $24 \mathrm{~h}$. The percentage of $\mathrm{LDH}$ released from injured astrocytes was significantly decreased when 1,000 and 3,000 U/ml of UTI were used. In a separate experiment, astrocytes were treated with UTI at a final concentration of $1,000 \mathrm{U} / \mathrm{ml}$ immediately, or at $30 \mathrm{~min}, 2,6$, or $24 \mathrm{~h}$ after sustained compression. The percentage of $\mathrm{LDH}$ release was significantly reduced $(P<0.05)$ when astrocytes were treated with UTI immediately or $30 \mathrm{~min}$ later. Together, our results suggest that UTI may have protective effects on astrocytes injured by sustained compression injury. Furthermore, the early administration ( $<2 \mathrm{~h}$ after injury) of UTI may result in a better outcome compared with delayed administration.
\end{abstract}

S. Zhang $\cdot$ R. Yu $(\bowtie) \cdot$ Y. Zhang $\cdot$ K. Chen

SICU, Fujian Provincial Hospital, Fujian Medical University Affiliated Provincial Teaching Hospital, Fuzhou 350001, China e-mail: garyyrg@yahoo.com
Keywords Astrocytes - Compression injury · LDH release $\cdot$ UTI $\cdot$ Cytoprotection

\section{Introduction}

Traumatic brain injury (TBI) is a significant cause of disability and death worldwide, and its incidence is rising sharply. Current medical therapies exhibit limited efficacy in reversing the initial damage and patients' prognosis remains poor [1], creating a significant burden for individuals, families and the society in general.

Early research on TBI focused almost exclusively on the injury to neurons with little attention paid to astrocytes. However, in the past decade, many new discoveries led to the recognition of a critical role for astrocytes in the outcome of TBI. Astrocytes, a sub-type of glial cells, are ubiquitous throughout all regions of the central nervous system (CNS). They outnumber neurons by more than fivefold and contiguously tile the entire CNS [1]. Normal astrocytes perform several functions, including: metabolic support, regulation of blood flow, formation of the blood brain barrier (BBB), transmitter uptake and release, modulation of synaptic transmission, and regulation of breathing [2-8]. Astrocytes are vulnerable to CNS insults and are severely damaged and die following TBI $[9,10]$. Subsequently, reactive astrogliosis occurs in surviving astrocytes. Reactive astrogliosis involves changes in gene expression, morphology and proliferation in a gradated fashion in relation to the severity of the injury [11-13]. Although the exact details of the role of reactive astrocytes are not completely understood, transgenic mice experiments revealed that ablation of reactive astrocytes after CNS injury causes substantial neuronal inflammation, degeneration, and death [14-16]. Therefore, astrocyte function is 
crucial for neurons, and affects outcomes following TBI, making astrocytes an important therapeutic target for neuronal protection following brain injury [17].

Damage to the integrity of cell membranes is the primary pathological change observed in mechanically injured cells [18]. It is a major contributor to the development of neuronal damage because it results in the increased accumulation of intracellular sodium, water and calcium ions, in leakage of enzymes and cofactors, and in the activation of cellular pathways [18]. Therefore we tested a therapeutic strategy targeting the protection of damaged cell membranes following TBI. Urinary trypsin inhibitor (UTI), one of the serine-type protease inhibitors isolated from human urine, inhibits not only a variety of proteases such as trypsin, $\alpha$-chymotrypsin, leukocyte elastases, and cathepsin G, but also hyaluronidase [19]. Several studies demonstrated that UTI may protect against systemic inflammation and reduce the injury induced by pancreatitis, sepsis and ischemia-reperfusion [20-22]. In CNS diseases, UTI may have therapeutic effects in craniocerebral injuries, since it decreases post-ischemic brain edema, oxidative stress, early inflammation and ischemia-reperfusion injury [23, 24]. However, UTI's protective effects against astrocyte membrane damage after TBI have not been reported. Our study demonstrates that UTI may have a cytoprotective effect on astrocytes injured by sustained compression injury.

\section{Materials and methods}

Ethics statement

All procedures followed the National Guide for the Care and Use of Laboratory Animals. All animal experiments were approved by the Fujian Provincial Hospital Committee on Ethics in the Care and Use of Laboratory Animals.

\section{Cell culture}

Astrocyte cultures were prepared using a previously described method $[25,26]$. Cortices were isolated from 1to 2-day-old rat pups, cleaned of white matter and meninges, minced, and digested with $0.25 \%$ trypsinEDTA (GIBCO, Invitrogen Inc., Carlsbad, CA, USA) for $20 \mathrm{~min}$ in a $37{ }^{\circ} \mathrm{C}$ water bath. Cells were next diluted into Dulbecco's modified Eagle medium/nutrient mixture F-12 (DMEM/F12, Hyclone, Thermo Fisher Scientific Inc., Waltham, MA, USA) supplemented with $10 \%$ fetal bovine serum (FBS, Hyclone, Thermo Fisher Scientific Inc., Waltham, MA, USA), washed, triturated, counted and seeded into $25-\mathrm{cm}^{2}$ flasks (NUNC, Thermo Fisher Scientific Inc., Waltham, MA, USA) at an initial density of
$0.5-1 \times 10^{6}$ cells per flask. Flasks were cultured at $37^{\circ} \mathrm{C}$ in $5 \% \mathrm{CO}_{2}$. The medium was changed every 2-3 days until culture attained confluence. To remove neurons, microglia and oligodendrocyte precursor cells, flasks were shaken at $250 \mathrm{rpm}$ for $18 \mathrm{~h}$ at $37{ }^{\circ} \mathrm{C}$ in a temperaturecontrolled orbital shaker (Shanghai Fuma Laboratory Equipment Co., Ltd., Shanghai, China). After medium removal, cells were trypsinized for 5-7 min. The trypsin was diluted with DMEM/F12 supplemented with $10 \%$ FBS, thus allowing the cells to lift from the flask bottom. The lifted cells were washed, centrifuged, counted, and seeded into $25-\mathrm{cm}^{2}$ flasks at a density of $0.5-1 \times 10^{6}$ cells per flask. Astrocyte cultures were used in experiments for a total of 4 weeks after removal from rats. Cultures consisted of at least $95 \%$ astrocytes as determined by glial fibrillary acidic protein (GFAP) immunocytochemistry.

\section{Cell injury}

Astrocytes for injury experiments were placed in 48-well $\left(1.1 \mathrm{~cm}^{2}\right.$ each) multidishes (NUNC, Thermo Fisher Scientific Inc., Waltham, MA, USA). When the cells reached a confluent monolayer, the DMEM/F12 was changed to serum-free medium 1 day before the experiment. Astrocytes were injured using a pressure device developed by Murphy and Horrocks [27]. Pressure was induced by increasing atmospheric pressure within a stainless steel chamber $(19.5 \mathrm{~cm}$ in length, $19.5 \mathrm{~cm}$ in width, and $20 \mathrm{~cm}$ in height). The chamber contained high-purity compressed air (78\% nitrogen, $21 \%$ oxygen, $0.93 \%$ argon, $0.04 \%$ carbon dioxide). Pressure duration time was 1 or $5 \mathrm{~min}$ with a pressure ranging from 0.1 to $0.3 \mathrm{MPa}$. The optimal duration time and pressure were determined based on the percentage of lactate dehydrogenase (LDH) released. Control cultures were incubated at $37{ }^{\circ} \mathrm{C}$ in $5 \% \mathrm{CO}_{2}$ during experimentation.

\section{UTI administration}

Astrocytes for experiments were placed in 48-well $\left(1.1 \mathrm{~cm}^{2}\right.$ each) multidishes. When the cells reached a confluent monolayer, the incubated medium for cells was changed to serum-free medium 1 day before the experiment. 0.3 MPa of pressure was administered to astrocytes for $5 \mathrm{~min}$. Subsequently, the injured cells were treated with UTI (Techpool Bio-Pharma Co. Ltd., Guangzhou, China), dissolved in serum-free culture medium at final concentrations of $100,1,000,3,000$ and 5,000 $\mathrm{U} / \mathrm{ml}$, and maintained for $24 \mathrm{~h}$.

Based on the results of the UTI intervention experiment, astrocytes plated in 48-well multidishes were randomly divided into 5 groups, according to the time of UTI exposure; each group comprised 3 subgroups: control 
group, injury group, and UTI intervention group. Cells in the UTI group were treated with UTI at a final concentration of $1,000 \mathrm{U} / \mathrm{ml}$ immediately, or at $30 \mathrm{~min}, 2 \mathrm{~h}, 6 \mathrm{~h}$, and $24 \mathrm{~h}$ after sustained compression (0.3 MPa, $5 \mathrm{~min})$.

\section{LDH release}

The degree of damage to cell membrane integrity has previously been evaluated by measuring the percentage of intracellular LDH released [18, 27-29]. LDH was measured using an in vitro toxicology assay kit (Sigma, St. Louis, MO, USA). The assay is based on the reduction reaction of nicotinamide adenine dinucleotide (NAD) by $\mathrm{LDH}$ [30]. The extracellular medium was sampled after the mechanical stimulus. The remaining intracellular LDH was also measured after solubilizing the cells with $0.2 \%$ Triton $\mathrm{X}-100$. The $\mathrm{LDH}$ release rate $(\%)$ was calculated using the following equation:

LDH release $(\%)=\frac{\text { Extracellurar LDH } \times 100}{\text { Intracellular LDH }+ \text { Extracellular LDH }}$

Transmission electron microscopic examination

Transmission electron microscope (TEM) was used to examine the ultrastructural details of injured astrocytes. Astrocytes were plated in 6-well $\left(9.6 \mathrm{~cm}^{2}\right.$ each well) multidishes (NUNC, Thermo Fisher Scientific Inc., Waltham, MA, USA). First, 0.3 MPa of pressure was administered to a monolayer of astrocytes for $5 \mathrm{~min}$. Next, each well was rinsed with phosphate-buffered saline (PBS, $\mathrm{pH}$ 7.4, GIBCO, Life Technologies Co., Waltham, MA, USA), and trypsinized for 4-5 min. The trypsinized cells were diluted with DMEM/F12 supplemented with $10 \%$ FBS. The lifted cells were centrifuged and fixed with $3 \%$ glutaraldehyde and $1.5 \%$ paraformaldehyde in PBS at room temperature for $4 \mathrm{~h}$. After being rinsed with PBS, samples were fixed with $1 \%$ osmic acid and $1.5 \%$ potassium ferrocyanide at $4{ }^{\circ} \mathrm{C}$ for $1.5 \mathrm{~h}$. Samples were then dehydrated with graded ethanol and acetone, embedded in epoxy resin, and cut into ultra-thin slices of about 70-80 nm. Slices were stained with uranyl acetate and lead citrate, and observed under an EM208 TEM (Philips, Royal Dutch Philips Electronics Ltd., Best, The Netherlands).

\section{Statistical analysis}

All data are presented as mean \pm standard deviation (SD). Statistical analyses were performed using the SPSS 13.0 software (SPSS Inc., Chicago, IL, USA). Statistical significance was established by one-way analysis of variance (ANOVA) followed by least significant difference (LSD) $t$ tests. $P$ values $<0.05$ were considered to be significant.

\section{Results}

Pressure-induced cell LDH release and morphological changes

No significant increase in the percentage of $\mathrm{LDH}$ release was detected after exposure to increased pressure for a short duration of time (1 min). When the duration of exposure was increased to $5 \mathrm{~min}$, a significant increase in $\mathrm{LDH}$ release from astrocytes was observed at a pressure of $0.3 \mathrm{MPa}$ (Fig. 1). Astrocyte morphology was studied using TEM. Uninjured astrocytes in the control group showed normal ultrastructural features (Fig. 2a). On the other hand, injured astrocytes displayed structural abnormalities, including endoplasmic reticulum swelling, mitochondrial cristae disruption and Golgi apparatus vacuolization (Fig. 2b). Therefore, in the following experiments, a pressure of $0.3 \mathrm{MPa}$ for $5 \mathrm{~min}$ was used to establish the sustained compression trauma model of astrocytes.

Protective effect of UTI on injured astrocytes

The percentage of LDH release increased significantly in injury samples (0.3 $\mathrm{MPa}, 5 \mathrm{~min})$ compared with the control samples. This result demonstrated that the astrocytes were injured by sustained compression. After being treated with UTI at concentrations of 1,000 and 3,000 U/ml for $24 \mathrm{~h}$, astrocytes released significantly less LDH compared with injury samples. However, a higher UTI concentration $(5,000 \mathrm{U} / \mathrm{ml})$ had no additional protective effect (Fig. 3).

In addition, the time-dependent effect of UTI administration on LDH release after injury was also studied. After being treated with $1,000 \mathrm{U} / \mathrm{ml}$ of UTI immediately, or 30 min post injury, astrocytes released significantly less LDH compared with untreated injury samples. The

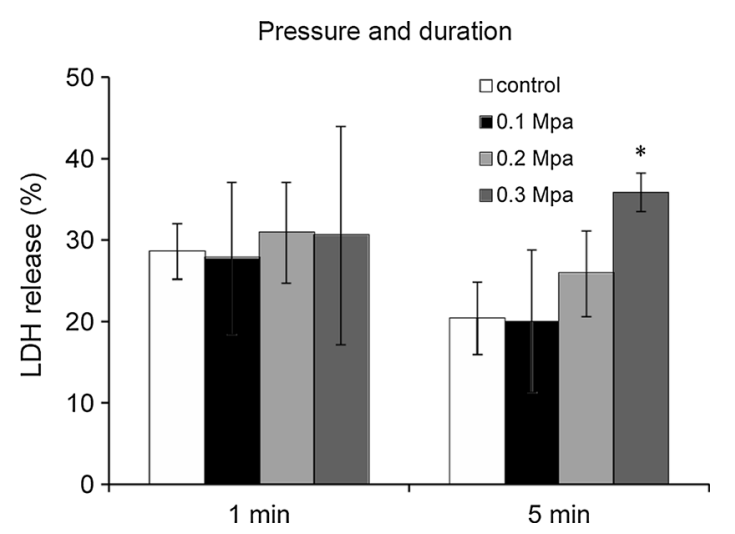

Fig. 1 Effects of sustained compression on LDH release from astrocytes. Pressure duration of 1 or $5 \mathrm{~min}$ was used on astrocytes over a pressure of $0.1,0.2$, or $0.3 \mathrm{MPa}$. LDH release was expressed as a percent of the total releasable LDH (extracellular and intracellular $\mathrm{LDH})$. Values are presented as mean $\pm \mathrm{SD}$. $* P<0.01$ versus control 

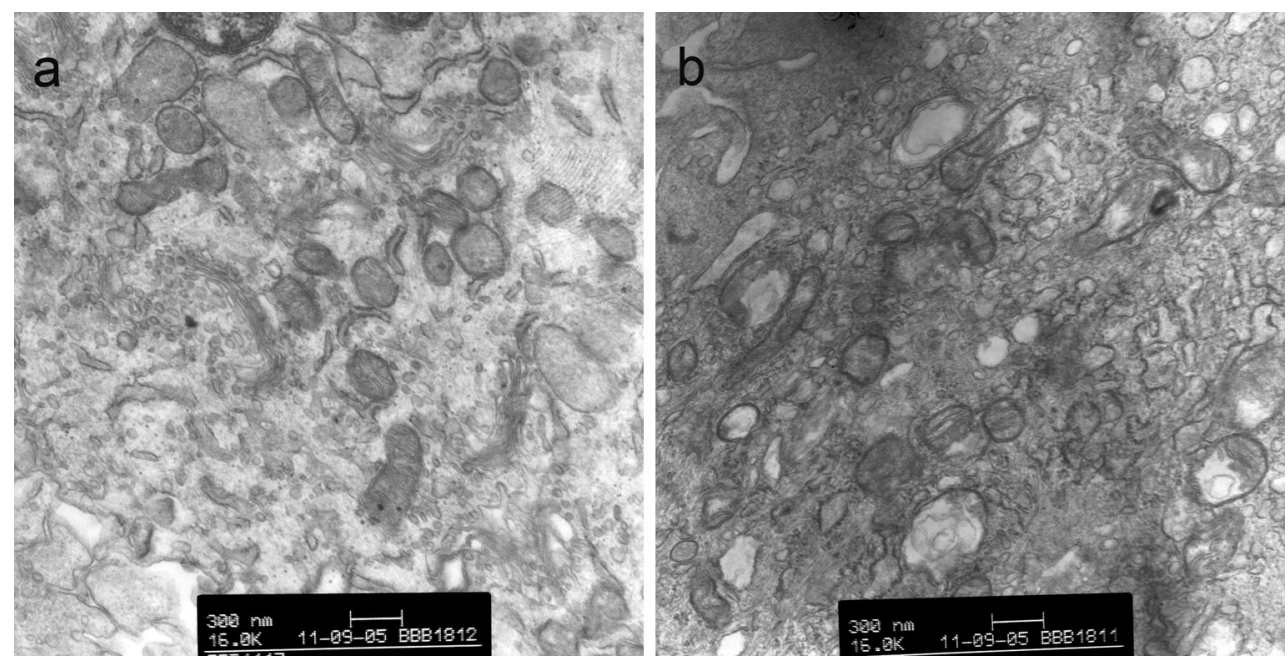

Fig. 2 Astrocyte morphology before and after sustained compression injury. a Normal ultrastructural features of uninjured astrocytes. b Ultrastructural abnormalities in astrocytes injured by sustained compression injury ( $0.3 \mathrm{MPa}$ for $5 \mathrm{~min}$ )

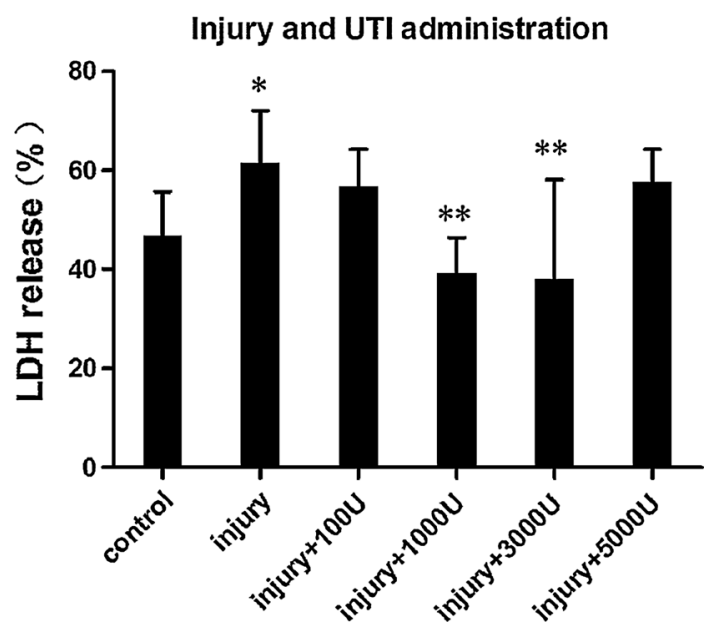

Fig. 3 Effects of different concentrations of UTI on LDH release from injured astrocytes. $* P<0.05$ versus control; $* * P<0.01$ versus injury. The amount and duration of pressure for the injury and UTI group were $0.3 \mathrm{MPa}$ and $5 \mathrm{~min}$. LDH release was expressed as a percent of the total releasable LDH (extracellular and intracellular $\mathrm{LDH})$. Values are presented as mean $\pm \mathrm{SD}$

protective effect was lost when astrocytes were treated with UTI $2 \mathrm{~h}$ post-injury. At later time points, the percentage of LDH released from injured astrocytes declined and no statistically significant differences in LDH release were detected among control, injury and UTI-treated group at 6 and $24 \mathrm{~h}$ post-injury (Fig. 4). This result demonstrated that UTI may have protective effects on astrocytes at the early stage of injury ( $<2 \mathrm{~h}$ after injury), and that membrane damage may be irreversible following an early window of recovery. Finally, our data also suggest that based on LDH release, astrocytes eventually recovered from pressureinduced injury in our model and that this recovery was unaffected by UTI.

\section{Discussion}

Using a hydrostatic pressure model of mechanical-like TBI, we studied the presumptive cytoprotective effects of UTI on injured astrocytes in vitro. Although in vitro brain injury models cannot surpass in vivo models, the former are able to complement the latter, explore different aspects, provide better control over experimental variables, and fulfill real time and spatial measurement of biological and mechanical parameters [31, 32]. The model employed in our research was a nonelastic chamber where a constant hydrostatic pressure was applied for a defined time, and compressed air was used as the gas source in order to eliminate potentially confounding factors such as ischemia and hypoxia. In cells subjected to a high-pressure environment, once the lateral compressions exceed the tolerance of cell membranes, cellular membrane integrity is compromised. According to the studies of Murphy and Horrocks [27], the effect of sustained hydrostatic pressure-induced injury depended on pressure and pressure duration, and these two parameters varied for different types of cells. Based on these results, we tested pressure durations of 1 and $5 \mathrm{~min}$ over a pressure range of $0.1,0.2$, and $0.3 \mathrm{MPa}$ on astrocytes. At a pressure of $0.3 \mathrm{MPa}$ during $5 \mathrm{~min}$, a significant increase in LDH released from astrocytes was observed. The increase in cytoplasmic enzyme efflux is an indicator of compromised cell membrane integrity. In addition, injured astrocytes displayed ultrastructural abnormalities, including endoplasmic reticulum swelling, mitochondrial cristae disruption and Golgi apparatus vacuolization, and these abnormalities were in accordance with an increased LDH release. These morphological abnormalities implied that certain cell functions may be impaired, such as the production and transport of proteins, energy conversion, and oxidative phosphorylation. These 


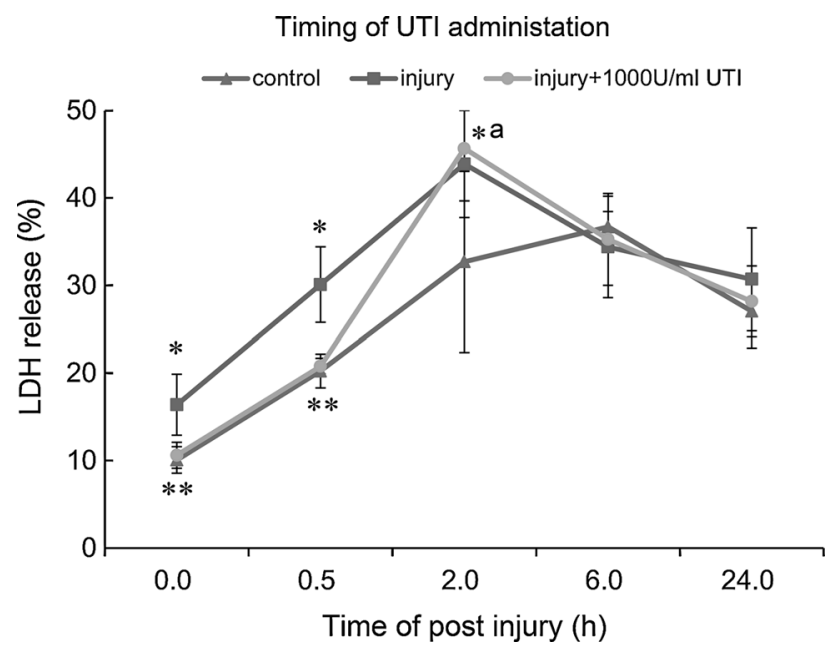

Fig. 4 Time-dependent effects of UTI on LDH release from injured astrocytes. ${ }^{*} P<0.01$ versus control; ${ }^{* a} P<0.05$ versus control; $* * P<0.01$ versus injury. The amount and duration of pressure for the injury and UTI groups were $0.3 \mathrm{MPa}$ and $5 \mathrm{~min}$. LDH release was expressed as a percent of the total releasable LDH (extracellular and intracellular LDH). Values are presented as mean \pm SD

results show that a hydrostatic pressure model of mechanical-like TBI on astrocytes was successfully established, and that the injured cells were characterized by decreased cell membrane integrity and ultrastructural abnormalities. Thus, LDH assay alone was used in our experiments.

Cell membrane integrity damage is a primary pathological change after mechanical neuronal injury. It is also the major contributor to the development of neuronal damage resulting in ionic imbalances and activation of several cellular pathways [18]. There are several ways to assess cell membrane integrity, including vital dye staining and measurement of cytoplasmic enzyme release. Vital dyes, such as trypan blue and propidium iodide (PI), are easily used and cheap, but they are usually time-consuming and do not allow the processing of a large number of samples. Furthermore, they do not account for the proportion of dead cells, and the actual rate of cell death may thus be underestimated [33]. In contrast, measurement of cytoplasm enzymes release is more accurate, easily automated, and reliably quantifies neuroprotective effects [34], and LDH is the most commonly assessed among these enzymes. Compared with other released enzymes (such as glucose-6-phosphate dehydrogenase, which has a half-life of $\sim 2 \mathrm{~h}$ in culture medium), LDH is more stable [35]. Moreover, the percentage of LDH released was used to characterize membrane damage instead of the amount of LDH released. Apart from cell membrane integrity, the amount of LDH efflux is also associated with the number of cells present. Therefore, the percentage of LDH release, a ratio of the amount of LDH efflux to the total LDH, was used to eliminate the influence of the number of cells on our quantification of LDH release.

Our studies demonstrated that the treatment of injured astrocytes with UTI at a concentration of 1,000 or $3,000 \mathrm{U} /$ $\mathrm{ml}$ significantly improved cell membrane integrity. In addition, the timing of UTI administration was also critical. The mechanism underlying the cytoprotective effects of UTI on injured astrocytes may involve the inhibition of hydrolytic enzymes released from lysosomes. Lysosomes are membrane-bound vesicles serving as the cell's main digestive compartment. Lysosomes contain numerous hydrolytic enzymes, including proteases, peptidases, phosphatases, nucleases, sulphatases and lipases. Together, these hydrolytic enzymes are capable of digesting all cellular components. In healthy cells, the lysosomal membrane protects cellular components from these degradative enzymes [36]. However, acute brain injury such as trauma, seizures and ischemia can result in the increase of free intracellular calcium and zinc. Calcium and zinc toxicity may contribute to lysosomal membrane permeabilization, disintegration and leakage of hydrolytic enzymes leading to the digestion of cellular components. This process is one of the major cascades involved in astrocyte necrosis [3741]. Lysosomal enzyme inhibitors may therefore be neuroprotective in brain injury. Being a serine-type protease inhibitor, UTI may protect cell proteins from digestion by inhibiting lysosomal proteases. In this way, UTI may stabilize the membrane of lysosomes and promote the resealing of disrupted cell membranes. However, increasing UTI dose to $5,000 \mathrm{U} / \mathrm{ml}$ had no further effect compared with the $3,000 \mathrm{U} / \mathrm{ml}$ dose. This may be due to the fact that UTI is an inhibitor of a variety of proteases and that high UTI doses may interfere with normal cellular proteolytic processes, thus counter-balancing its beneficial effects.

In conclusion, UTI may have cytoprotective effects on astrocytes injured by sustained compression trauma due to the improvement of cell membrane integrity. Timedependent treatment is essential as the early administration ( $<2 \mathrm{~h}$ after injury) of UTI resulted in better protection compared with the delayed administration.

Acknowledgments This study was supported by grants from the National Clinical Key Specialty (2011170) and the Tianpu Corporation (01200914). We are grateful to Dr. Ling Lin and Dr. Zhihong Zheng (Fujian Medical University) for instruction on astrocytes culture, and to Zhenfei Peng and Jingdi Chen (Life Science Institute of Fuzhou University, China) for offering their assistance.

Conflict of interest The authors declare that they have no conflict of interest.

Open Access This article is distributed under the terms of the Creative Commons Attribution License which permits any use, distribution, and reproduction in any medium, provided the original author(s) and the source are credited. 


\section{References}

1. Laird MD, Vender JR, Dhandapani KM (2008) Opposing roles for reactive astrocytes following traumatic brain injury. Neurosignals 16:154-164

2. Koehler RC, Roman RJ, Harder DR (2009) Astrocytes and the regulation of cerebral blood flow. Trends Neurosci 32:160-169

3. Attwell D, Buchan AM, Charpak S, Lauritzen M, Macvicar BA, Newman EA (2010) Glial and neuronal control of brain blood flow. Nature 468:232-243

4. Brown AM, Ransom BR (2007) Astrocyte glycogen and brain energy metabolism. Glia 55:1263-1271

5. Correale J, Villa A (2009) Cellular elements of the blood-brain barrier. Neurochem Res 34:2067-2077

6. Halassa MM, Fellin T, Haydon PG (2007) The tripartite synapse: roles for gliotransmission in health and disease. Trends Mol Med 13:54-63

7. Perea G, Navarrete M, Araque A (2009) Tripartite synapses: astrocytes process and control synaptic information. Trends Neurosci 32:421-431

8. Gourine AV, Kasymov V, Marina N, Tang F, Figueiredo MF, Lane $\mathrm{S}$ et al (2010) Astrocytes control breathing through $\mathrm{pH}-$ dependent release of ATP. Science 329:571-575

9. Liu D, Smith CL, Barone FC, Ellison JA, Lysko PG, Li K et al (1999) Astrocytic demise precedes delayed neuronal death in focal ischemic rat brain. Brain Res Mol Brain Res 68:29-41

10. Zhao X, Ahram A, Berman RF, Muizelaar JP, Lyeth BG (2003) Early loss of astrocytes after experimental traumatic brain injury. Glia 44:140-152

11. Chen Y, Swanson RA (2003) Astrocytes and brain injury. J Cereb Blood Flow Metab 23:137-149

12. Danilov CA, Fiskum G (2008) Hyperoxia promotes astrocyte cell death after oxygen and glucose deprivation. Glia 56:801-808

13. Sofroniew MV, Vinters HV (2010) Astrocytes: biology and pathology. Acta Neuropathol 119:7-35

14. Faulkner JR, Herrmann JE, Woo MJ, Tansey KE, Doan NB, Sofroniew MV (2004) Reactive astrocytes protect tissue and preserve function after spinal cord injury. J Neurosci 24:2143-2155

15. Myer DJ, Gurkoff GG, Lee SM, Hovda DA, Sofroniew MV (2006) Essential protective roles of reactive astrocytes in traumatic brain injury. Brain 129:2761-2772

16. Voskuhl RR, Peterson RS, Song B, Ao Y, Morales LB, TiwariWoodruff S et al (2009) Reactive astrocytes form scar-like perivascular barriers to leukocytes during adaptive immune inflammation of the CNS. J Neurosci 29:11511-11522

17. Barreto GE, Gonzalez J, Torres Y, Morales L (2011) Astrocyticneuronal crosstalk: implications for neuroprotection from brain injury. Neurosci Res 71:107-113

18. Serbest G, Horwitz J, Barbee K (2005) The effect of poloxamer188 on neuronal cell recovery from mechanical injury. J Neurotrauma 22:119-132

19. Nakahama H, Kuribayashi K, Sugita M, Shinkawa T (1999) Cytoprotective effect of ulinastatin, a Kunitz-type protease inhibitor, on hypoxic injury in L2 cells treated with antimycin A via stabilization of lysosomal fragility. Pulm Pharmacol Ther 12:1-6

20. Yano T, Anraku S, Nakayama R, Ushijima K (2003) Neuroprotective effect of urinary trypsin inhibitor against focal cerebral ischemia-reperfusion injury in rats. Anesthesiology 98:465-473

21. Cao YZ, Tu YY, Chen X, Wang BL, Zhong YX, Liu MH (2012) Protective effect of Ulinastatin against murine models of sepsis: inhibition of TNF-alpha and IL-6 and augmentation of IL-10 and IL-13. Exp Toxicol Pathol 64:543-547

22. Nduaguibe CC, Bentsi-Barnes K, Mullen Y, Kandeel F, Al-Abdullah I (2010) Serine protease inhibitors suppress pancreatic endogenous proteases and modulate bacterial neutral proteases. Islets 2:200-206

23. Du HG, Yin LC, He M, Zhang GJ, Tian Y, Wang C et al (2005) Application of ulinastatin in severe craniocerebral injuries. Chin $\mathrm{J}$ Traumatol 8:236-239

24. Koga Y, Fujita M, Tsuruta R, Koda Y, Nakahara T, Yagi T et al (2010) Urinary trypsin inhibitor suppresses excessive superoxide anion radical generation in blood, oxidative stress, early inflammation, and endothelial injury in forebrain ischemia/reperfusion rats. Neurol Res 32:925-932

25. Ahmed SM, Rzigalinski BA, Willoughby KA, Sitterding HA, Ellis EF (2000) Stretch-induced injury alters mitochondrial membrane potential and cellular ATP in cultured astrocytes and neurons. J Neurochem 74:1951-1960

26. Taylor AR, Robinson MB, Milligan CE (2007) In vitro methods to prepare astrocyte and motoneuron cultures for the investigation of potential in vivo interactions. Nat Protoc 2:1499-1507

27. Murphy EJ, Horrocks LA (1993) A model for compression trauma: pressure-induced injury in cell cultures. J Neurotrauma $10: 431-444$

28. Ellis EF, McKinney JS, Willoughby KA, Liang S, Povlishock JT (1995) A new model for rapid stretch-induced injury of cells in culture: characterization of the model using astrocytes. J Neurotrauma 12:325-339

29. Frieboes LR, Gupta R (2009) An in vitro traumatic model to evaluate the response of myelinated cultures to sustained hydrostatic compression injury. J Neurotrauma 26:2245-2256

30. Decker T, Lohmann-Matthes ML (1988) A quick and simple method for the quantitation of lactate dehydrogenase release in measurements of cellular cytotoxicity and tumor necrosis factor (TNF) activity. J Immunol Methods 115:61-69

31. Morrison B III, Saatman KE, Meaney DF, McIntosh TK (1998) In vitro central nervous system models of mechanically induced trauma: a review. J Neurotrauma 15:911-928

32. Kumaria A, Tolias CM (2008) In vitro models of neurotrauma. Br J Neurosurg 22:200-206

33. Giordano G, Hong S, Faustman EM, Costa LG (2011) Measurements of cell death in neuronal and glial cells. Methods Mol Biol 758:171-178

34. Lobner D (2000) Comparison of the LDH and MTT assays for quantifying cell death: validity for neuronal apoptosis? J Neurosci Methods 96:147-152

35. Riss TL, Moravec RA (2004) Use of multiple assay endpoints to investigate the effects of incubation time, dose of toxin, and plating density in cell-based cytotoxicity assays. Assay Drug Dev Technol 2:51-62

36. Yamashima T, Oikawa $S$ (2009) The role of lysosomal rupture in neuronal death. Prog Neurobiol 89:343-358

37. Raghupathi R (2004) Cell death mechanisms following traumatic brain injury. Brain Pathol 14:215-222

38. Farkas O, Povlishock JT (2007) Cellular and subcellular change evoked by diffuse traumatic brain injury: a complex web of change extending far beyond focal damage. Prog Brain Res 161:43-59

39. Dressler J, Hanisch U, Kuhlisch E, Geiger KD (2007) Neuronal and glial apoptosis in human traumatic brain injury. Int $\mathrm{J}$ Legal Med 121:365-375

40. Lee SJ, Koh JY (2010) Roles of zinc and metallothionein-3 in oxidative stress-induced lysosomal dysfunction, cell death, and autophagy in neurons and astrocytes. Mol Brain 3:30

41. McAllister TW (2011) Neurobiological consequences of traumatic brain injury. Dialogues Clin Neurosci 13:287-300 